\title{
Declaración Sociedad Chilena de Enfermedades Respiratorias en relación a regulación del mentol en los cigarrillos
}

\author{
Statement of the Chilean Society of Respiratory Diseases on mentholated cigarettes
}

En febrero de 2005, entró en vigencia el Convenio Marco para el Control del Tabaco $(\mathrm{CMCT})^{1}$, el primer tratado mundial de salud de la OMS cuyo objetivo es "proteger a las generaciones presentes y futuras contra las devastadoras consecuencias sanitarias, sociales, ambientales y económicas producto del consumo de tabaco y de la exposición al humo de tabaco". Es un acuerdo de carácter vinculante para los países que lo suscriban y ratifiquen y expresa la voluntad política de las naciones de apoyar las medidas propuestas.

Chile suscribe el Convenio en septiembre de 2003 y el Parlamento lo ratifica en junio de 2005. En mayo de 2006, se promulgó la modificación a la Ley del Tabaco (20.105) que entró en vigencia en forma paulatina a partir del 16 de agosto de ese mismo año ${ }^{2}$. Este convenio fue realizado por expertos en derecho internacional, que entienden que no es necesaria evidencia local para tomar decisiones en legislación de la salud, por esto el CMCT y sus directrices son aplicables a todas las naciones.

Según la Encuesta Nacional de Salud de Chile 2009-2010 3 , se observa una elevada prevalencia de tabaquismo en población adulta $(40,6 \%)$ lo que constituye uno de los más altos niveles de consumo a nivel latinoamericano, consumo que se ha mantenido elevado desde 2003. Adicionalmente, se detectó un incremento en el número de cigarrillos promedio diarios consumidos $(10,4$ cigarrillos/día). El nivel de dependencia a la nicotina, es de $33,2 \%$ entre los fumadores, cifra muy preocupante ya que estos fumadores necesitan ayuda médica para dejar de fumar, asistencia actualmente no garantizada.

En relación a la evidencia científica que avala la prohibición del uso del mentol, es necesario aclarar que no es factible realizar estudios orientados a demostrar relación de causa-efecto entre el mentol que contiene el tabaco y determinados efectos en salud, puesto que en el humo de tabaco, el mentol se combina con más de 7.000 otros compuestos, siendo imposible aislar sus efectos.
La evidencia científica disponible, se basa en estudios epidemiológicos de gran calidad y precisión, que relacionan al mentol como químico independiente del cigarrillo con otros factores de riesgo, es la metodología utilizada en la mayoría de las investigaciones científicas para tomar decisiones en relación a problemas de salud a nivel mundial. Un ejemplo de ellos es, el proceso de implementación del CMCT en las diferentes legislaciones. Dicha metodología ha conformado la base de evidencia científica que ha permitido legislar para el control del consumo y exposición al humo ambiental de tabaco (Chile, 2006, 2012).

El mentol de los productos de tabaco tiene entre sus múltiples efectos farmacológicos, el de enmascarar el efecto irritante del humo de tabaco, por lo que es más fácil de inhalar, aumentando el potencial de adicción y de este modo el riesgo de cáncer ${ }^{4}$.

Contribuye también a aumentar el atractivo de productos inherentemente perjudiciales y adictivos entre los consumidores, especialmente entre los jóvenes y los nuevos fumadores. Hay consenso general de que el atractivo de los productos tóxicos del tabaco debe ser significativamente reducido a través del Convenio Marco de la OMS para el Control del tabaco (CMCT) La directriz del CMCT artículo 9 establece que las medidas dirigidas a reducir el atractivo de los productos de tabaco pueden contribuir a reducir la prevalencia del consumo de tabaco y la dependencia entre los usuarios nuevos y con experiencia.

El artículo 9 también exige medidas eficaces para regular los contenidos de los productos del tabaco, estableciendo lo siguiente: "Desde la perspectiva de la salud pública, no hay justificación para permitir el uso de ingredientes, tales como agentes aromatizantes, que ayudan a hacer del tabaco productos atractivos. [ ... ]. El atractivo y su impacto en la dependencia deben tenerse en cuenta al considerar las medidas reglamentarias. [...]. Enmascarar la dureza humo del tabaco con sabores contribuye a promover y sostener el con- 
sumo de tabaco". Las directrices recomiendan la prohibición de los ingredientes que mejoran el sabor de los productos del tabaco, ya que aumentan el atractivo de los productos nocivos y por lo tanto, al final favorecen la aparición de la adicción.

El Comité Científico Asesor de Productos de Tabaco (TPSAC) de los EE.UU. Food and Drug Administration (FDA) ${ }^{5}$ concluyó en un detallado y reciente informe sobre los cigarrillos mentolados y la salud pública "Que el mentol tiene efectos refrescantes y anestésicos que reducen la dureza del humo del cigarrillo. La investigación indica que existe acción del mentol en receptores térmicos y nociceptivos Esta es una doble acción de refrigeración del humo y disminución de sus efectos irritantes. Las implicancias de estos hallazgos son que al reducir la dureza del humo del tabaco mentolado podría facilitar la iniciación o la persistencia temprana del consumo de tabaco entre los jóvenes. Además, mediante la reducción de la dureza de humo, es biológicamente posible que el mentol facilitara la inhalación más profunda y prolongada de humo de tabaco, lo que resulta en mayor exposición de humo de cigarrillo".

Los países donde el uso de cigarrillos mentolados está muy extendido y los datos están disponibles, muestran que el mentol de los cigarrillos atrae especialmente a los jóvenes: El TPSAC hace la recomendación general a la FDA, que "La eliminación de los cigarrillos mentolados del mercado beneficiaría la salud pública en los Estados Unidos". Esto se basó en el aumento relativo del consumo de cigarrillos de mentol entre los jóvenes.

Por estas razones, el mentol no puede considerarse meramente un aditivo saborizante al tabaco. Sus acciones farmacológicas reducen la aspereza del humo y la irritación de la nicotina, e incrementa la probabilidad de adicción a la nicotina en los adolescentes y adultos jóvenes que experimentan con el tabaquismo. Por otra parte, las características sensoriales del mentol pueden aumentar la adicción -de los cigarrillos mentolados, que parece ser el caso de los jóvenes.

TPSAC ha encontrado que la disponibilidad de cigarrillos de mentol tiene un efecto negativo en la salud pública al aumentar el número de fumadores con resultado de muerte prematura y morbilidad evitable ${ }^{6}$.

Chile presenta la mayor prevalencia de fumadoras adolescentes entre 13 y 15 años a nivel mundial, la mayor prevalencia de adolescentes fumadores entre 13 y 15 años en Latinoamericano y la mayor prevalencia de fumadores en población general en nuestro continente latinoamericano.

Por estas razones, la Sociedad Chilena de Enfermedades Respiratorias se hace un deber en manifestar que, la incorporación de medidas que restrinjan el uso de aditivos en los productos del tabaco, deben ser adoptadas a la brevedad y sin dilación. Ellas son parte de un acuerdo y compromiso internacional que nuestro país firmó y ratificó, y cuya finalidad es exclusivamente el beneficio de toda la población del país y de las futuras generaciones.

Drs. María Paz Corvalán B. y Gonzalo Valdivia $\boldsymbol{C}$.

Comisión de Tabaquismo, Sociedad Chilena de Enfermedades Respiratorias.

\section{Bibliografía}

1.- CMCT. http://www.who.int/fctc/es

2.- VALENZUELA SCHMIDT MT (2010) Chile: Situación del tabaquismo a cinco años de la ratificación del Convenio Marco para el Control del Tabaco y los desafíos pendientes Informe de Chile Libre de Tabaco. http:// www.chilelibredetabaco.cl/descargas/Informe_Chile_5_anos_CMCT_EPES_2010.pdf

3.- ENS 2012. http://epi.minsal.cl/estudios-y-encuestas-poblacionales/encuestas-poblacionales/encuesta-nacionalde-salud/

4.- German Cancer Research Center (2012) Menthol Capsules in Cigarette Filters - Increasing the Attractiveness of a Harmful Product. Volume 17, Red Series Tobacco Prevention and Tobacco Control, http://www.dkfz.de/ de/tabakkontrolle/download/Publikationen/RoteReihe/ Band_17_Menthol_Capsules_in_Cigarette_Filters en.pdf

5.- Tobacco Products Scientific Advisory Committee (2011) Menthol Cigarettes and Public Health: Review of the Scientific Evidence and Recommendations. Submitted to FDA: March 23, 2011, final edits from the July 21, 2011 meeting are included, http://www.fda.gov/downloads/AdvisoryCommittees/CommitteesMeetingMaterials/TobaccoProductsScientificAdvisoryCommittee/ UCM269697.pdf

6.- Tobacco Products Scientific Advisory Committee (2011) Menthol Cigarettes and Public Health: Review of the Scientific Evidence and Recommendations. Submitted to FDA: March 23, 2011, final edits from the July 21, 2011 meeting are included, http://www.fda.gov/downloads/AdvisoryCommittees/CommitteesMeetingMaterials/TobaccoProductsScientificAdvisoryCommittee/ UCM269697.pdf 